\title{
Evaluación técnica y económica del pepino y el pimentón como alternativas al tomate bajo invernadero
}

\section{Technical and economic evaluation of cucumber and pepper crops as alternatives to greenhouse tomato production}
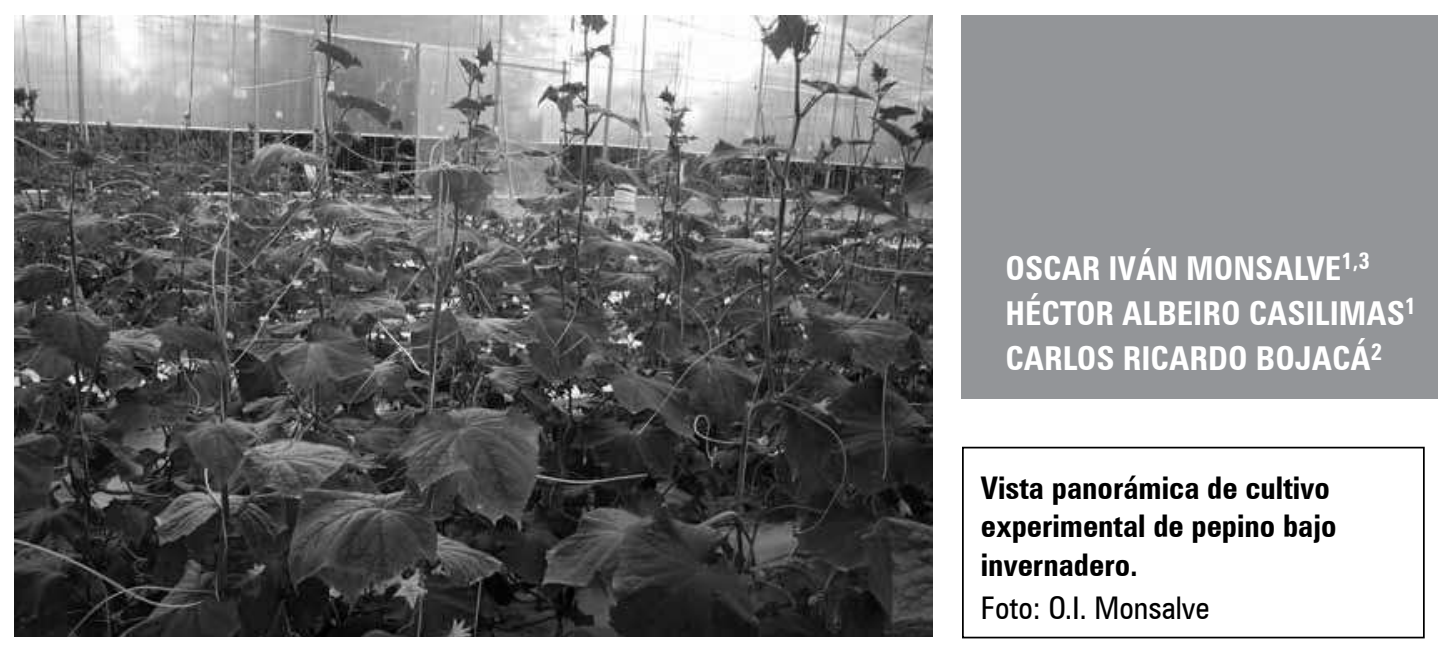

\section{RESUMEN}

Con el objetivo de encontrar alternativas de diversificación al tomate, se realizó una evaluación agronómica y económica de cinco híbridos de pepino (Cucumis sativus L.) y trece de pimentón (Capsicum annuum L.), mediante el establecimiento de cultivos semicomerciales. La evaluación económica comparó cinco proyectos: tomate larga vida, pepino cohombro y europeo, pimentón rojo y gourmet; a través de tres escenarios comerciales. Los dos primeros consideraron el comportamiento financiero ante variaciones de precio y productividad (desde -50 hasta 50\% con respecto al valor de referencia). El tercero analizó los proyectos cuando el precio pagado varió en función del cliente. La evaluación agronómica no mostró diferencias significativas para los híbridos de pepino europeo y pimentón gourmet. Para cada cultivo, el pepino cohombro Caman, el pepino europeo Azabache y el pimentón gourmet Taranto presentaron las mayores producciones con 8,0; 8,5 y 7,9 $\mathrm{kg} \mathrm{m}^{-2}$, respectivamente. Los híbridos de pimentón rojo mostraron diferencias significativas, siendo AF6529 con $4,8 \mathrm{~kg} \mathrm{~m}^{-2}$ el que presentó la mayor productividad. La evaluación económica demostró la viabilidad de los proyectos de pimentón gourmet, tomate larga vida y pepino europeo, siendo el pimentón gourmet el de mayores retornos, pues con una inversión de \$187.552.083 recupera la inversión en dos años, obteniendo la rentabilidad anual deseada y una ganancia extra de $\$ 60.909 .707$. El incremento en productividad de pepino cohombro y pimentón rojo es necesario para aumentar su competitividad; sin embargo, si se logra obtener un producto de alta calidad y volúmenes constantes, es posible acceder a mercados de mayor rentabilidad.

1 Facultad de Ciencias Naturales e Ingeniería, Centro de Bio-Sistemas, Universidad de Bogotá Jorge Tadeo Lozano, Bogotá (Colombia).

2 Facultad de Ciencias Naturales e Ingeniería, Departamento de Ciencias Básicas, Universidad de Bogotá Jorge Tadeo Lozano, Bogotá (Colombia).

3 Autor para correspondencia. oscar.monsalve@utadeo.edu.co 


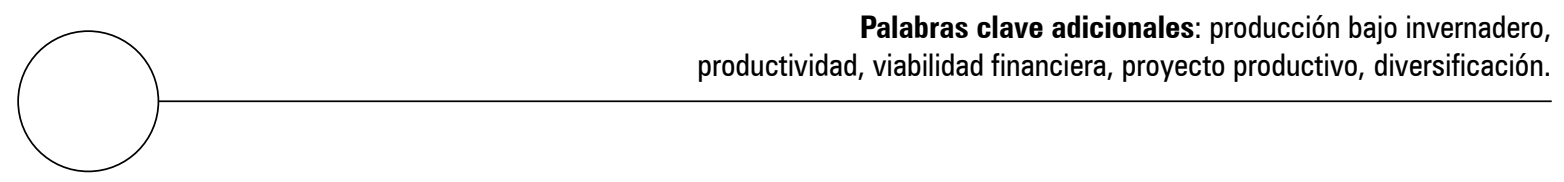

\section{ABSTRACT}

In order to find alternatives to greenhouse tomato production, a technical and economic evaluation of five cucumber (Cucumis sativus L.) hybrids and thirteen sweet pepper (Capsicum annumm L.) hybrids was carried out using semi-commercial crops. The economic evaluation compared five projects: long shelf-life tomato, regular and European cucumber, and red and gourmet sweet pepper, using three different commercial scenarios. The first two considered the financial results when price and productivity variations (from -50 up to $50 \%$ with respect to the reference value) were applied. The third one analyzed price paid variations as a function of the customer. For each crop, the highest production came from the regular cucumber Caman, the European cucumber Azabache and the gourmet sweet pepper Taranto with $8.0,8.5$ and $7.9 \mathrm{~kg} \mathrm{~m}^{-2}$, respectively. The red sweet pepper hybrids showed significant differences, with AF6529 displaying the highest productivity with $4.8 \mathrm{~kg} \mathrm{~m}^{-2}$. The economic evaluation indicated that the gourmet sweet pepper, long shelflife tomato and European cucumber are feasible projects, with the gourmet sweet pepper project having the highest returns. The initial investment of COP $\$ 187.552 .083$ is recovered in two years, obtaining the desired profitability while generating a surplus of COP $\$ 60.909 .707$. It is necessary to increase the productivity of the regular cucumber and the red sweet pepper in order to make them more competitive in wholesale markets; however, if the quality of the products is improved while offering constant volumes, it is possible to gain access to more profitable markets.

Additional key words: greenhouse production, productivity, financial feasibility, productive project, diversification.

Fecha de recepción: 02-03-2011

Aprobado para publicación: 27-05-2011
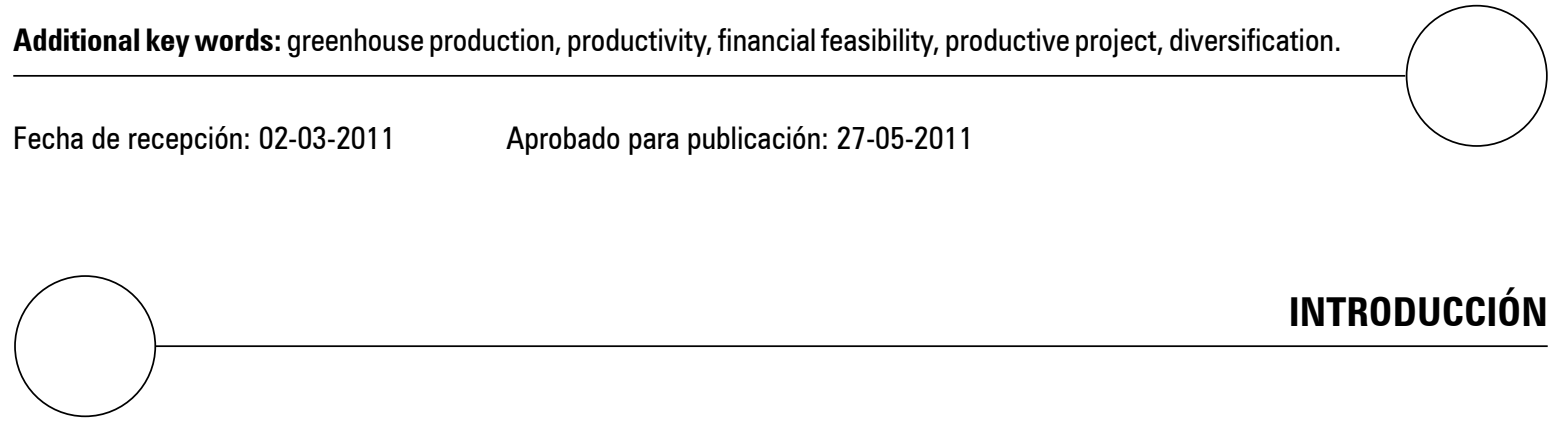

INTRODUCCIÓN

El tomate (Solanum lycopersicum L.) es la principal hortaliza cultivada bajo condiciones de invernadero en Colombia. Las estadísticas sobre el área cultivada bajo este sistema productivo no son consistentes y los valores reportados varían entre 500 ha (Jaramillo, 2009) y 3.391 ha (Miranda et al., 2009), siendo Cundinamarca, Norte de Santander, Valle de Cauca, Boyacá, Huila, Antioquia, Risaralda y Caldas los principales departamentos productores. Aunque el desarrollo de este sistema productivo ha permitido incrementar los rendimientos y proteger los cultivos de las inclemencias del clima (Bojacá et al., 2009a; Jaramillo, 2009), la producción de esta hortaliza afronta de manera permanente otra serie de riesgos. Factores como la constante variación de precios pagados al productor, de los rendimientos debida a la heterogeneidad de prácticas de manejo aplicadas (Bojacá et al., 2009b) y la competencia comercial con el tomate producido a campo 
abierto generan un desequilibrio permanente en la cadena productiva, así como incertidumbres sobre la rentabilidad real de este cultivo.

Aunque los invernaderos utilizados en Colombia se caracterizan por ser estructuras de bajo costo y con mínimas opciones de control del clima (Bojacá et al., 2011), la permanencia del cultivo de tomate bajo invernadero en el tiempo ha demostrado su capacidad para pagar los costos derivados de la instalación y mantenimiento de dicha infraestructura. De acuerdo con la Corporación Colombia Internacional (2006) la instalación y mantenimiento del invernadero representan el $32,1 \%$ de los costos totales. Aún cuando para Escobar (2009) este ítem represente el 13\%, es innegable que los costos de esta infraestructura son un factor importante a la hora de establecer sistemas productivos bajo condiciones protegidas.

Aparte del tomate, las hortalizas más cultivadas bajo invernadero a nivel mundial son el pepino (Cucumis sativus L.), el pimentón (Capsicum annuum L.) y la berenjena (Solanun melongena L.), tanto en condiciones de suelo como bajo hidroponía (Bunte, 2009; Castilla y Montero, 2008; Mohammadi y Omid, 2010; Ozkan, 2004). El establecimiento de estos sistemas productivos ha demostrado ser viable tanto técnica como económicamente. La producción bajo condiciones protegidas ofrece una rentabilidad mayor a la de las producciones a campo abierto para estos productos de acuerdo con lo expuesto por Pozderec et al. (2010) quienes lo demostraron empleando herramientas de modelado aplicadas a los cultivos de pepino y pimentón.

El trabajo realizado por Mohammadi y Omid (2010) consistente en evaluar tanto ambiental como económicamente la producción de pepino bajo invernadero en Irán demuestra que bajo las condiciones locales de producción fue posible obtener una relación beneficio-costo igual a 2,58 a partir de una productividad de $11,9 \mathrm{~kg} \mathrm{~m}^{-2}$. Aunque este trabajo no hace mayor referencia al manejo agronómico empleado, si hace referencia a la utilización de sistemas de calefacción dentro de los invernaderos para optimizar las condiciones de producción. En Turquía, la producción hidropónica de pepino bajo invernadero logró una relación beneficio-costo de 1,53 con base en una productividad de $32,3 \mathrm{~kg} \mathrm{~m}^{-2}$ (Engindeniz y Gül, 2009). En este caso el invernadero consistió en una estructura metálica empleando polietileno como material de cubierta.

Singh y Sirohi (2006) reconociendo el potencial que tenía el establecimiento de invernaderos semi-climatizados en India evaluaron diferentes variedades de pimentones de colores obteniendo producciones entre las 40 y $50 \mathrm{t} \mathrm{ha}^{-1}$. Sin embargo los mismos autores reconocen que la viabilidad económica de estos sistemas depende directamente del tipo de mercado disponible para el productor. El análisis económico realizado por Gunadi et al. (2007) sobre la producción de pimentón en invernaderos plásticos en Indonesia reveló la rentabilidad positiva de este sistema donde se alcanzó una relación beneficio-costo de 2,12 cuando el riego se realizaba por goteo o de 2,03 cuando era manual.

El tomate al ser considerado la única hortaliza viable para ser establecida bajo condiciones de invernadero aumenta el riesgo financiero al cual se ven sometidos los productores. Es necesario considerar alternativas de diversificación que resulten atractivas para los productores tanto en lo técnico como en lo económico. Sin embargo, para nuestras condiciones locales de producción no se dispone de información validada experimentalmente que permita realizar un proceso adecuado de toma de decisiones. Reportes sobre la caracterización agronómica de los cultivos de pepino y pimentón bajo las condiciones de invernadero aplicadas en Colombia son casi inexistentes. En el caso de pepino, únicamente se reporta una evaluación del comportamiento agronómico de cinco cultivares de pepino cohombro (Arjona et al., 1992), mientras que para pimentón la búsqueda bibliográfica realizada no arrojó ningún resultado. 
A partir de en lo anterior y ante la ausencia de información actualizada, el objetivo del presente trabajo fue evaluar la viabilidad técnica y financiera del establecimiento de los cultivos de pepino y pimentón como alternativas al actual sistema productivo de tomate bajo condiciones protegidas. Los objetivos específicos consistieron en caracterizar agronómicamente diferentes materiales híbridos tanto de pepino como de pimentón y, establecer el efecto de variaciones de precios, productividad y destino de la producción sobre los indicadores de viabilidad empleados en la evaluación económica.

\section{MATERIALES Y MÉTODOS}

Esta investigación se llevó a cabo en el Centro de Bio-Sistemas de la Universidad Jorge Tadeo Lozano de Bogotá, ubicado en el municipio de Chía (Cundinamarca) $\left(4^{\circ} 53^{\prime} 3,62^{\prime \prime} \mathrm{N},-74^{\circ} 00^{\prime} 50^{\prime \prime} \mathrm{O}\right)$ a una altura de $2.650 \mathrm{msnm}$. Para este estudio se dispuso un invernadero de vidrio tipo Venlo con un área de $1.000 \mathrm{~m}^{2}$, en el cual se evaluó la producción de tres materiales de pepino cohombro y dos materiales de pepino europeo (tabla 1), siete variedades de pimentón tipo gourmet y seis variedades de pimentón rojo (tabla 2) durante un ciclo de cultivo.

\section{Evaluación agronómica}

El invernadero se dividió en dos secciones de 500 $\mathrm{m}^{2}$ mediante barreras plásticas. En cada medio invernadero se establecieron los cultivos experimentales de pepino y pimentón de forma tal que todos los híbridos estuviesen incluidos en la evaluación.

En el caso del pepino, el área experimental contuvo 14 camas de cultivo con una dimensión de 0,9 m de ancho por $24 \mathrm{~m}$ de largo. Cada cama fue dividida en cinco unidades experimentales (UE) de $5 \mathrm{~m}^{2}$ que incluían siete plantas por cada híbrido. Los híbridos se trasplantaron a una densidad de 1,4 plantas/m. La evaluación se realizó entre el 13 de marzo del 2009 y el 18 de agosto del mismo año, con una duración de 23 semanas. Durante este ciclo productivo se registró una temperatura promedio de $16^{\circ} \mathrm{C}$ y una humedad relativa (HR) promedio de $90,5 \%$. Se implementó el mismo manejo cultural, nutricional y sanitario para todos los híbridos evaluados, empleando para ello criterios de buenas prácticas agrícolas.

La evaluación de los materiales de pimentones se dividió en dos grupos; el primero compuesto por los materiales gourmet, que tienen frutos de colores amarillos, naranjas y mentas y están dirigidos a mercados especializados, y el segundo grupo con los materiales que tienen frutos de color rojo, los cuales se encuentran comúnmente en el mercado nacional. Cada área experimental contenía doce camas, con una dimensión de 0,8 $\mathrm{m}$ de ancho por $24 \mathrm{~m}$ de largo. Los materiales se trasplantaron a una densidad de 2,3 plantas $/ \mathrm{m}$. Cada UE tuvo un área de $7 \mathrm{~m}^{2}$ con 16 plantas

Tabla 1. Productividad y número de días a cosecha de los híbridos de pepino cohombro y europeo (partenocárpico) evaluados bajo condiciones semicomerciales.

\begin{tabular}{|l|l|l|c|c|}
\hline Híbrido & \multicolumn{1}{|c|}{ Tipo } & Empresa & Productividad $\left(\mathrm{kg} \mathrm{m}^{-2}\right)$ & Trasplante a cosecha (días) \\
\hline Azabache & Partenocárpico & Enza Zaden & $8,5 \mathrm{a}$ & $91 \mathrm{a}$ \\
\hline 3053 & Partenocárpico & Western Seed & $8,1 \mathrm{a}$ & $91 \mathrm{a}$ \\
\hline Adrian & Cohombro & Rijk Zwaan & $6,4 \mathrm{~b}$ & $90 \mathrm{a}$ \\
\hline Caman & Cohombro & Rijk Zwaan & $7,9 \mathrm{a}$ & $87 \mathrm{a}$ \\
\hline Tornado & Cohombro & Nirit Seeds & $6,0 \mathrm{~b}$ & $86 \mathrm{a}$ \\
\hline
\end{tabular}

Promedios con letras distintas para cada variable y tipo de pepino indican diferencia significativa según la prueba de Tukey $(P \leq 0,05)$. 
Tabla 2. Productividad y número de días a cosecha de los híbridos de pimentón gourmet y rojo evaluados bajo condiciones semicomerciales.

\begin{tabular}{|c|c|c|c|c|c|}
\hline Híbrido & Tipo & Empresa & Color & Productividad (kg m²) & Trasplante a Cosecha (días) \\
\hline Lirica & Gourmet & Rijk Zwaan & Naranja & $7,5 \mathrm{a}$ & 239 e \\
\hline Menta & Gourmet & Rijk Zwaan & Verde & $7,3 \mathrm{a}$ & $177 \mathrm{a}$ \\
\hline Plinio & Gourmet & Rijk Zwaan & Amarillo & $6,8 \mathrm{a}$ & $216 d$ \\
\hline Orangery & Gourmet & Rijk Zwaan & Naranja & $7,4 \mathrm{a}$ & $218 d$ \\
\hline Taranto & Gourmet & Rijk Zwaan & Amarillo & $7,9 \mathrm{a}$ & $218 d$ \\
\hline Zamboni & Gourmet & Rijk Zwaan & Morado & 6,9 a & $203 c$ \\
\hline Zirconio & Gourmet & Rijk Zwaan & Morado & $6,9 \mathrm{a}$ & $184 a b$ \\
\hline AF6529 & Rojo & Rijk Zwaan & Rojo & $4,8 \mathrm{a}$ & $188 \mathrm{ab}$ \\
\hline Brenan & Rojo & Rijk Zwaan & Rojo & $3,1 \mathrm{~d}$ & $213 d$ \\
\hline Brito F1 & Rojo & Rijk Zwaan & Rojo & $3,1 \mathrm{~cd}$ & $190 \mathrm{~b}$ \\
\hline Dhara & Rojo & Rijk Zwaan & Rojo & $3,9 \mathrm{bc}$ & $185 a$ \\
\hline Magaly & Rojo & Rijk Zwaan & Rojo & $4,1 a b$ & 197 c \\
\hline Nathalie & Rojo & $\begin{array}{l}\text { Roger } \\
\text { Seeds }\end{array}$ & Rojo & $4,3 a b$ & $204 d$ \\
\hline
\end{tabular}

Promedios con letras distintas para cada variable y tipo de pimentón indican diferencia significativa según la prueba de Tukey $(P \leq 0,05)$.

por cada material. La evaluación de los materiales de pimentón gourmet y rojos se realizó entre el 21 de abril del 2009 hasta el 29 de noviembre del mismo año, con una duración de 31 semanas. Durante el ciclo de cultivo se presentó una temperatura promedio de $16,2^{\circ} \mathrm{C}$ y una $\mathrm{HR}$ promedio de $89 \%$. Al igual que en el caso del pepino, durante el periodo productivo se implementó el mismo manejo cultural, nutricional y sanitario para todos los materiales evaluados, atendiendo criterios de buenas prácticas agrícolas.

El diseño experimental bajo el cual se realizaron las evaluaciones de híbridos tanto de pepino como de pimentón consistió en un diseño completamente al azar con cuatro repeticiones por híbrido en las cuales se evaluó semanalmente la producción de frutos en términos de kg por planta y $\mathrm{kg} \mathrm{m}^{-2}$ y la precocidad de cada híbrido, medido en días a cosecha. El análisis estadístico de los datos consistió en la realización de análisis de varianza con el fin de establecer la existencia o no de diferencias significativas entre las productividades de los diferentes híbridos en evaluación para cada cultivo. Posteriormente se aplicó la prueba de comparaciones múltiples de Tukey con el fin de establecer posibles agrupamientos entre las medias de los diferentes híbridos de cada cultivo. Estos análisis se realizaron empleando el paquete de análisis estadístico R (R Development Core Team, 2011). Durante los distintos ciclos de cultivo se llevó un registro detallado de todos los insumos utilizados así como de la mano de obra empleada para el sostenimiento de los cultivos. Esta información fue procesada para su posterior uso en la evaluación económica.

\section{Evaluación económica}

La evaluación económica se basó en un modelo empresarial, en donde todos los procesos tanto técnicos como administrativos y de gestión se caracterizan por estar dentro de los parámetros legales para el gobierno colombiano (CCB, 2011; DIAN, 2011). Los proyectos productivos analizados fueron pepino cohombro (PEC), pepino europeo (PEE), pimentón gourmet (PIG), pimentón rojo $(P I R)$ y tomate larga vida $(T L V)$. Todos los datos técnicos relativos a fertilización, protección de cultivos, labores culturales, cosecha, poscosecha, entre otros, fueron cuantificados económicamente e incluidos en la matriz de da- 
tos de entrada para la evaluación económica. De igual manera, todos los costos variables (material vegetal, fertilizantes, protección de cultivos y papelería) y fijos (salarios y administración) asociados a la producción fueron contabilizados e incluidos en el análisis. La fuente de los datos de $T L V$ correspondió a una encuesta aplicada a 170 productores ubicados en los municipios de Sáchica, Santa Sofía, Sutamarchán, Tinjacá y Villa de Leyva (Boyacá). El objetivo de esta encuesta fue caracterizar a los productores de tomate bajo invernadero ubicados en estos municipios y la información recogida permitió extraer los datos necesarios para realizar la evaluación económica.

Los principales parámetros técnicos y económicos tenidos en cuenta para la evaluación econó- mica de cada uno de los proyectos productivos se encuentran expuestos en la tabla 3. El análisis asumió que la producción sería constante para un área de cultivo de 1 ha en cada uno de los proyectos evaluados. La evaluación económica de todos los proyectos consideró una tasa de impuestos del 33\% (DIAN, 2011), una vida útil de activos depreciables de 5 años, una tasa para los depósitos a término fijo (DTF) del 4\%, una tasa de interés del 12\% (Finagro, 2011) para un préstamo solicitado a 2 años y una tasa de inflación anual del 3\% (Banco de la República, 2011). El proyecto fue evaluado de tal forma que el productor obtuviera una rentabilidad deseada del $21 \%$, considerando una tasa de oportunidad del $24 \%$, la cuál se obtuvo de la suma entre la rentabilidad deseada más la inflación estimada para

Tabla 3. Parámetros iniciales tomados en cuenta para el análisis financiero de los cinco proyectos productivos evaluados.

\begin{tabular}{|c|c|c|c|c|c|}
\hline Parámetro & Tomate & $\begin{array}{l}\text { Pepino } \\
\text { cohombro }\end{array}$ & $\begin{array}{c}\text { Pepino } \\
\text { partenocárpico }\end{array}$ & $\begin{array}{l}\text { Pimentón } \\
\text { rojo }\end{array}$ & $\begin{array}{l}\text { Pimentón } \\
\text { gourmet }\end{array}$ \\
\hline \multicolumn{6}{|l|}{ Datos técnicos } \\
\hline Área de cultivo $\left(\mathrm{m}^{2}\right)$ & 10.000 & 10.000 & 10.000 & 10.000 & 10.000 \\
\hline Área en producción constante $\left(\mathrm{m}^{2}\right)$ & 5.000 & 6.667 & 6.667 & 5.000 & 5.000 \\
\hline Densidad (plantas $/ \mathrm{m}^{2}$ ) & 2,7 & 1,4 & 1,4 & 2,5 & 2,5 \\
\hline Productividad por planta (kg/planta y ciclo) & 5,0 & 5,8 & 6,4 & 1,9 & 3,2 \\
\hline Productividad por area $\left(\mathrm{kg} \mathrm{m}^{-2}\right.$ y ciclo) & 13,5 & 8,1 & 9,0 & 4,8 & 8,0 \\
\hline Productividad anual $\left(\mathrm{kg} \mathrm{m}^{-2} \mathrm{año}^{-1}\right)$ & 27,0 & 16,2 & 17,9 & 7,1 & 12,0 \\
\hline Producción mensual $\left(\mathrm{kg} \mathrm{mes}^{-1}\right)$ & 22.500 & 13.533 & 14.933 & 5.938 & 10.000 \\
\hline Duración ciclo (meses) & 6 & 6 & 6 & 8 & 8 \\
\hline \multicolumn{6}{|l|}{ Salarios } \\
\hline Salario administrador $\left(\$\right.$ mes $\left.^{-1}\right)$ & 1.200 .000 & 1.200 .000 & 1.200 .000 & 1.200 .000 & 1.200 .000 \\
\hline Salario operario jornales $\left(\$\right.$ mes $\left.^{-1}\right)$ & 600.000 & 600.000 & 600.000 & 600.000 & 600.000 \\
\hline Salario contador $\left(\$\right.$ mes $\left.^{-1}\right)$ & 250.000 & 250.000 & 250.000 & 250.000 & 250.000 \\
\hline \multicolumn{6}{|l|}{ Precios pagados al productor } \\
\hline Abastos $\left(\$ \mathrm{~kg}^{-1}\right)$ & 1.128 & 825 & 825 & 1.681 & 1.681 \\
\hline Gran superficie $\left(\$ \mathrm{~kg}^{-1}\right)$ & 1.692 & 1.237 & 1.237 & 2.521 & 2.521 \\
\hline Exportador $\left(\$ \mathrm{~kg}^{-1}\right)$ & 1.297 & 949 & 949 & 1.933 & 1.933 \\
\hline Tienda $\left(\$ \mathrm{~kg}^{-1}\right)$ & 1.410 & 1.031 & 1.031 & 2.101 & 2.101 \\
\hline Consumidor final tipo $1\left(\$ \mathrm{~kg}^{-1}\right)$ & 1.833 & 1.340 & 1.340 & 2.731 & 2.731 \\
\hline Consumidor final tipo $2\left(\$ \mathrm{~kg}^{-1}\right)$ & 2.820 & 2.062 & 2.062 & 4.202 & 4.202 \\
\hline
\end{tabular}

REV. COLOMB. CIENC. HORTIC. 
los cinco años del proyecto (3\%). La rentabilidad deseada se definió a partir de experiencias prácticas en campo que indican que esta oscila entre 21 y $23 \%$ para proyectos agrícolas sostenibles. A partir de esta información y como indicador financiero se calculó el valor presente neto (VPN), definido de acuerdo a la siguiente fórmula:

$V P N=\frac{F C_{1}}{(1+i)^{1}}+\frac{F C_{2}}{(1+i)^{2}}+\frac{F C_{n}}{(1+i)^{n}}-\mathrm{VP}$

donde $F C$ representa el flujo de caja en los momentos indicados, $i$ es la tasa de descuento de los $F C$ equivalente a la tasa de retorno requerida por el proyecto, $n$ representa la vida del proyecto expresada según el número de periodos y $V P$ el valor presente de la inversión inicial.

Otro indicador financiero utilizado para evaluar los proyectos fue la tasa interna de retorno $(T I R)$, calculada a partir de la fórmula para el $V P N$, donde se asume que el VPN es igual a cero y que la variable a despejar es la tasa de interés $i$. También se calcularon la cantidad (PEU = Punto de equilibrio por unidades) y el precio de equilibrio $(P E V)$, así como la relación beneficio-costo $(B / C)$, de acuerdo con las siguientes fórmulas:

$$
\begin{aligned}
& P E U=\frac{\text { Costos fijos }}{\text { Precio de venta unitario - Costo variable unitario }} \\
& P E V=\frac{\text { Costos fijos }}{1-\frac{\text { Costos variables totales }}{\text { Ventas totales }}}
\end{aligned}
$$$$
B / C=\frac{\text { Valor presente de los ingresos }}{\text { Valor presente de los costos }}
$$

Aparte de realizar una evaluación económica fija para cada proyecto productivo se elaboraron distintos escenarios comerciales con el fin de establecer la viabilidad económica de cada proyecto ante variaciones de precio, productividad o punto de venta. Para tal fin se configuraron los siguientes escenarios comerciales:
Escenario 1: dado que los principales puntos de comercialización de la producción agrícola nacional son los centros mayoristas de abastos, los cuales se caracterizan por generar una fluctuación constante en los precios tanto de venta al consumidor como de compra al productor, se simuló el comportamiento financiero de los proyectos productivos ante aumentos y disminuciones de hasta el $50 \%$ del precio por kg pagado al productor. El precio de referencia a partir del cual se consideraron las fluctuaciones fue el precio promedio pagado al productor en la Corporación de Abastos de Bogotá (Corabastos) durante los últimos cinco años (los precios fueron corregidos por la inflación anual y traídos a valor presente). Esta información fue obtenida a través de los boletines del Sistema de Información de Precios del Sector Agropecuario (Sipsa).

Escenario 2: una de las características que define la producción bajo invernadero en Colombia es la falta de estandarización de prácticas de manejo. Lo anterior sumado a la diversidad de zonas donde se produce en condiciones protegidas genera comportamientos variables de la productividad. En consecuencia el segundo escenario consistió en establecer el efecto de variaciones tanto positivas como negativas de hasta un 50\% de la productividad para cada cultivo en términos de $\mathrm{kg} \mathrm{m}^{-2}$ vendido en los centros mayoristas de abastecimiento. La productividad utilizada como referencia correspondió a la productividad promedio de cada uno de los tipos de cultivos evaluados experimentalmente, y en el caso de tomate correspondió a la productividad promedio reportada por los agricultores en la encuesta.

Escenario 3: en el mercado nacional existen diferentes tipos de compradores a los cuales el productor bajo invernadero puede ofrecer su producto. Con el fin de analizar el impacto de la selección del comprador se comparó el comportamiento financiero de los cinco proyectos productivos ante diferentes tipos de clientes: Abastos, gran superficie, exportadora, tienda, supermercado o plaza de barrio, consumidor tipo 1 (estrato $\leq 3$ ) y consumidor tipo 2 (estrato $>3$ ). 
Las características de cada uno de los clientes potenciales, incluidas en la tabla 4 , incluyeron el número de compradores representado en la cantidad de compradores dentro de cada tipo de cliente; por ejemplo, el tomate de un solo productor se le vende a varias tiendas o supermercados de barrio, o le vende a un solo almacén de cadena. De igual forma se incluyeron características como la calidad exigida por cada tipo de cliente, las categorías de compra de cada cliente, el manejo del precio de compra, el manejo del volumen de compra, la frecuencia en la realización de los pagos, el tipo de empaque que exige cada cliente, el manejo de las devoluciones de producto deteriorado o dañado y la logística requerida para el transporte y la entrega de pedidos.

La explicación detallada de cada cliente se presenta en la tabla 4. La información de cada cliente, así como los precios de venta productor-cliente se establecieron con base en comunicaciones directas con los tipos de clientes relacionados.

\section{RESULTADOS Y DISCUSIÓN}

\section{Evaluación agronómica}

Pepino cohombro: los resultados de las evaluaciones de pepino se presentan en la tabla 1. El híbrido Caman muestra el mayor rendimiento, con una producción de $7,9 \mathrm{~kg} \mathrm{~m}^{-2}$. Al comparar el tiempo que los híbridos tardan en iniciar la producción desde trasplante, no se encontraron diferencias significativas. A pesar de esto, el híbrido Caman presentó la mayor precocidad, demorando en promedio $87 \mathrm{~d}$ desde trasplante a inicio de cosecha. Estos resultados demuestran que el híbrido Caman presenta las mejores condiciones para la producción intensiva bajo invernadero, ya que obtuvo el mayor rendimiento y fue uno de los más rápidos al momento de iniciar la producción.

Pepino europeo: no se encontraron diferencias significativas para el rendimiento de los materiales Azabachey 3053, que alcanzaron en promedio una producción de 8,5 y $8,1 \mathrm{~kg} \mathrm{~m}^{-2}$, respectivamente (tabla 1). Resultados similares se obtuvieron al analizar el tiempo que los híbridos tardan en iniciar cosecha, encontrándose un promedio de 91 días. Estos resultados demuestran que ambos híbridos de pepino europeo presentan buenas condiciones para ser producidos bajo invernadero.

Pimentón gourmet: la tabla 2 presenta los resultados de las evaluaciones semicomerciales realizadas con los híbridos de pimentón. Los resultados indicaron que no hubo diferencias significativas en la producción de los siete híbridos evaluados; sin embargo, el híbrido Taranto registró la mayor producción, con 7,9 $\mathrm{kg} \mathrm{m}^{-2}$, seguido de Lirica y Orangery. Al evaluar el tiempo que se demoran en llegar a producción desde trasplante, Menta fue el híbrido más precoz demorando 177 días en iniciar la producción, mientras que el material Lirica fue el más tardío (tabla 2).

Pimentón rojo: el híbrido AF6529 obtuvo la mayor producción, con $4,8 \mathrm{~kg} \mathrm{~m}^{-2}$ seguido de Nathalie con $4,3 \mathrm{~kg} \mathrm{~m}^{-2}$ y Magaly con $4,1 \mathrm{~kg}$ $\mathrm{m}^{-2}$. De otro lado, el híbrido Brenan presentó la producción más baja. De igual forma, el híbrido AF6529 presentó un menor periodo de tiempo para iniciar cosecha, demorando $188 \mathrm{~d}$ desde trasplante, seguido de Magaly, con 197 d y Nathalie con 204 d. Estos resultados muestran al híbrido AF6529 con las mejores características para cultivar bajo invernadero, ya que fue el más productivo y el más rápido para iniciar la producción (tabla 2).

\section{Evaluación económica}

De acuerdo a los resultados expuestos en la tabla 5 se puede apreciar que la producción de TLV, PIG y PEE son viables bajo la metodología y parámetros económicos aplicados. El proyecto PIG es el que mayores retornos económicos otorga al productor, pues con una inversión de \$187.552.083 recupera la inversión en dos años, obtiene la rentabilidad anual deseada (21\%) y además genera un superávit de \$60.909.707. 
Al igual que el proyecto PIG, el proyecto TLV también alcanza la rentabilidad anual deseada y genera beneficios económicos por encima de lo esperado (\$32.166.105) pero en menor proporción al proyecto PIG. Los proyectos PIR y PEC no son viables financieramente, manejados dentro del marco empresarial exigido por las Cámaras de Comercio y la DIAN, pues no se obtiene la rentabilidad esperada. El panorama más difícil lo presenta el proyecto $P I R$, al mostrar una rentabilidad negativa, lo que indica que los ingresos no alcanzan a cubrir los costos de producción ni a saldar la deuda adquirida para la inversión. Esto se debe principalmente a la baja producción $(4,8$ $\mathrm{kg} \mathrm{m}^{-2}$ ) en comparación con la producción del proyecto PIG $\left(8 \mathrm{~kg} \mathrm{~m}^{-2}\right)$. El proyecto PEC presenta una rentabilidad positiva de $16,7 \%$ pero no alcanza la rentabilidad esperada (20\%), lo que lo hace un proyecto no viable. El resultado anterior es debido a los bajos precios de venta utilizados para el análisis. A continuación se presentan los resultados obtenidos para las simulaciones de los escenarios comerciales de cada proyecto ante variaciones de precio, productividad o punto de venta.

Escenario 1: la figura 1 muestra que con las condiciones expuestas en el estudio y a excepción

\section{Tabla 4. Principales características de los diferentes tipos de clientes incluidos en la evaluación económica según} los datos recopilados en campo y conversación directa con productores y clientes.

\begin{tabular}{|c|c|c|c|c|c|c|}
\hline Característica & Abastos & $\begin{array}{c}\text { Gran } \\
\text { superficie }\end{array}$ & Exportador & Tienda & $\begin{array}{l}\text { Consumidor } \\
\text { tipo } 1\end{array}$ & $\begin{array}{c}\text { Consumidor } \\
\text { tipo } 2\end{array}$ \\
\hline $\begin{array}{l}\text { Distribución } \\
\text { del producto }\end{array}$ & $\begin{array}{l}\text { En punto de } \\
\text { venta }\end{array}$ & $\begin{array}{l}\text { En punto de } \\
\text { venta }\end{array}$ & $\begin{array}{l}\text { En punto de } \\
\text { venta }\end{array}$ & $\begin{array}{l}\text { En punto de } \\
\text { venta }\end{array}$ & $\begin{array}{l}\text { En punto de } \\
\text { venta }\end{array}$ & $\begin{array}{l}\text { En punto de } \\
\text { venta }\end{array}$ \\
\hline Transporte & Alquilado & Alquilado & Alquilado & Propio & Propio & Propio \\
\hline $\begin{array}{l}\text { Número } \\
\text { compradores }\end{array}$ & $\begin{array}{l}\text { Un único } \\
\text { comprador }\end{array}$ & $\begin{array}{l}\text { Un único } \\
\text { comprador }\end{array}$ & $\begin{array}{l}\text { Un único } \\
\text { comprador }\end{array}$ & $\begin{array}{l}\text { Varios } \\
\text { compradores }\end{array}$ & $\begin{array}{l}\text { Varios } \\
\text { compradores }\end{array}$ & $\begin{array}{l}\text { Varios } \\
\text { compradores }\end{array}$ \\
\hline $\begin{array}{l}\text { Exigencia de } \\
\text { calidad }\end{array}$ & Media & Alta & Alta & Media - Alta & Alta & Alta \\
\hline $\begin{array}{l}\text { Categorías de } \\
\text { compra }\end{array}$ & Todas & $\begin{array}{l}\text { Las dos } \\
\text { mejores }\end{array}$ & $\begin{array}{l}\text { Las dos } \\
\text { mejores }\end{array}$ & $\begin{array}{l}\text { Una o dos } \\
\text { categorías }\end{array}$ & $\begin{array}{l}\text { Una o dos } \\
\text { categorías }\end{array}$ & $\begin{array}{l}\text { Una o dos } \\
\text { categorías }\end{array}$ \\
\hline $\begin{array}{l}\text { Cantidad de } \\
\text { compra }\end{array}$ & Todo & Todo & Todo & Una parte & $\begin{array}{l}\text { Una pequeña } \\
\text { parte }\end{array}$ & $\begin{array}{l}\text { Una pequeña } \\
\text { parte }\end{array}$ \\
\hline $\begin{array}{l}\text { Precio de } \\
\text { compra }\end{array}$ & $\begin{array}{l}\text { Dependiendo } \\
\text { de la oferta del } \\
\text { producto en el } \\
\text { momento de la } \\
\text { transacción }\end{array}$ & $\begin{array}{l}20-200 \%> \\
\text { a Abastos }\end{array}$ & $\begin{array}{l}20-50 \%> \\
\text { a Abastos }\end{array}$ & $\begin{array}{l}\text { Igual al precio } \\
\text { de venta } \\
\text { (Abastos - } \\
\text { consumidor) }\end{array}$ & $\begin{array}{l}\text { Igual al precio } \\
\text { de venta } \\
\text { (tienda - } \\
\text { consumidor) }\end{array}$ & $\begin{array}{l}\text { Igual al precio } \\
\text { de venta } \\
\text { (tienda - } \\
\text { consumidor) }\end{array}$ \\
\hline $\begin{array}{l}\text { Volumen de } \\
\text { compra }\end{array}$ & Todo & Constante & $\begin{array}{l}\text { Constante } 0 \\
\text { por pedido }\end{array}$ & Constante & Por pedido & Por pedido \\
\hline $\begin{array}{l}\text { Frecuencia } \\
\text { pago }\end{array}$ & Contado & 15 - 60 días & 15 - 60 días & 0 - 15 días & Contado & Contado \\
\hline Empaque & Granel & Empacado & Empacado & Granel & Empacado & Empacado \\
\hline $\begin{array}{l}\text { Devoluciones } \\
\text { y/o daños }\end{array}$ & Abastos & $\begin{array}{l}\text { Productor } 0 \\
\text { Almacén }\end{array}$ & Productor & Tienda & Consumidor & Consumidor \\
\hline $\begin{array}{l}\text { Exigencia de } \\
\text { logística }\end{array}$ & Baja & Baja & Baja & Media & Alta & Alta \\
\hline
\end{tabular}


Tabla 5. Resultados financieros simulando la transacción comercial al precio promedio pagado al productor durante los últimos cinco años en la Corporación de Abastos de Bogotá (Corabastos) de acuerdo con los boletines del Sistema de Información de Precios del Sector Agropecuario (Sipsa).

\begin{tabular}{|c|c|c|c|c|c|}
\hline Parámetro & Tomate & $\begin{array}{l}\text { Pepino } \\
\text { cohombro }\end{array}$ & $\begin{array}{c}\text { Pepino } \\
\text { partenocárpico }\end{array}$ & Pimentón rojo & $\begin{array}{l}\text { Pimentón } \\
\text { gourmet }\end{array}$ \\
\hline Costos variables anuales $(\$)$ & 23.692 .000 & 10.706 .400 & 10.706 .400 & 19.548 .000 & 19.548 .000 \\
\hline Costos fijos anuales (\$) & 127.471 .500 & 94.961 .900 & 66.725 .400 & 68.543 .750 & 73.662 .500 \\
\hline Costos totales anuales $(\$)$ & 151.163 .500 & 105.668 .300 & 77.431 .800 & 88.091 .750 & 93.210 .500 \\
\hline Activos depreciables (\$) & 100.750 .000 & 99.853 .333 & 99.993 .333 & 99.093 .750 & 99.500 .000 \\
\hline Activos no depreciables (\$) & 104.553 .000 & 64.387 .133 & 65.563 .133 & 83.786 .458 & 88.052 .083 \\
\hline Inversión (\$) & 205.303 .000 & 164.240 .467 & 165.556 .467 & 182.880 .208 & 187.552 .083 \\
\hline Valor de salvamento (\$) & 30.225 .000 & 29.956 .000 & 29.998 .000 & 29.728 .125 & 29.850 .000 \\
\hline Préstamo (\$) & 164.242 .400 & 131.392 .373 & 132.445 .173 & 146.304 .167 & 150.041 .667 \\
\hline Valor presente neto (VPN, \$) & 32.166 .105 & -10.726 .441 & 9.934 .491 & -73.906 .343 & 60.909 .707 \\
\hline Tasa interna de retorno $(T I R, \%)$ & 40,8 & 16,7 & 30,1 & $-18,1$ & 60,2 \\
\hline Relación beneficio-costo $(B / C)$ & 1,2 & 1,0 & 1,1 & 0,8 & 1,3 \\
\hline Punto de equilibrio (kg/año) & 200.176 & 128.419 & 130.075 & 74.420 & 73.067 \\
\hline Precio de equilibrio $\left(\$ \mathrm{~kg}^{-1}\right)$ & 710 & 665 & 614 & 1.744 & 1.086 \\
\hline
\end{tabular}

del proyecto PIR, todos los cultivos presentan una TIR positiva. Sin embargo, solo los proyectos TLV, PIG y PEE presentan un VPN positivo. El indicador financiero VPN solo comienza a ser positivo para los proyectos PEC y PIR si se aumentan los precios de venta 10 y $40 \%$, respectivamente. Solo el proyecto PIG resiste una disminución máxima del $10 \%$ en el precio de venta para continuar siendo viable, al considerar tanto la $T I R$ como el VPN. El proyecto TLV presenta una pendiente mayor a los demás cultivos, debido a la mayor producción en términos de $\mathrm{kg} \mathrm{m}^{-2}$. Esto indica que una pequeña variación porcentual en el precio de venta ejerce un aumento o disminución notable tanto en la TIR como en el $V P N$, lo que no ocurre con los demás proyectos, los cuales necesitan una diferencia porcentual mayor para que la TIR y el VPN fluctúen considerablemente. Esto implica que para el proyecto $T L V$ la fluctuación de precios del mercado afecte en mayor proporción la rentabilidad del cultivo, lo que crea bastante inestabilidad en el proceso comercial.
Escenario 2: el comportamiento de la fluctuación porcentual en la producción de los proyectos en evaluación presenta una tendencia similar a lo expuesto en el escenario 1, pues los proyectos TLV, PIG y PEE muestran una TIR y un VPN positivo mientras que los proyectos $P E C$ y $P I R$ son inviables económicamente para las condiciones del estudio. Sería necesario un aumento de 10 y $40 \%$ en la producción $\left(\mathrm{kg} \mathrm{m}^{-2}\right)$ para que los proyectos $P E C$ y $P I R$, respectivamente, fuesen viables económicamente. Esto evidencia, que si se aumenta la producción en campo es posible enfrentar la inestabilidad comercial que afrontan los proyectos en estudio y generaría una rentabilidad estable en el tiempo. La experiencia en campo indica que es más factible mejorar las condiciones de cultivo y de esta forma generar una mayor productividad, que cambiar las políticas agrícolas comerciales en el país.

Escenario 3: la figura 3 muestra que cuando los clientes de los productores son las grandes superficies o los consumidores finales todos los 
proyectos son viables. La venta a las tiendas, supermercados y plazas de barrio es viable para todos los proyectos, excepto para PIR, debido a la baja producción $\left(\mathrm{kg} \mathrm{m}^{-2}\right)$ que tiene. La viabilidad económica de los proyectos PIG, TLV y PEE se ratifica con este análisis, pues con cualquier tipo de cliente los proyectos son rentables. Esta simulación muestra claramente que aun cuando la producción no sea óptima, si se realizan acuerdos comerciales con canales de distribución dife-

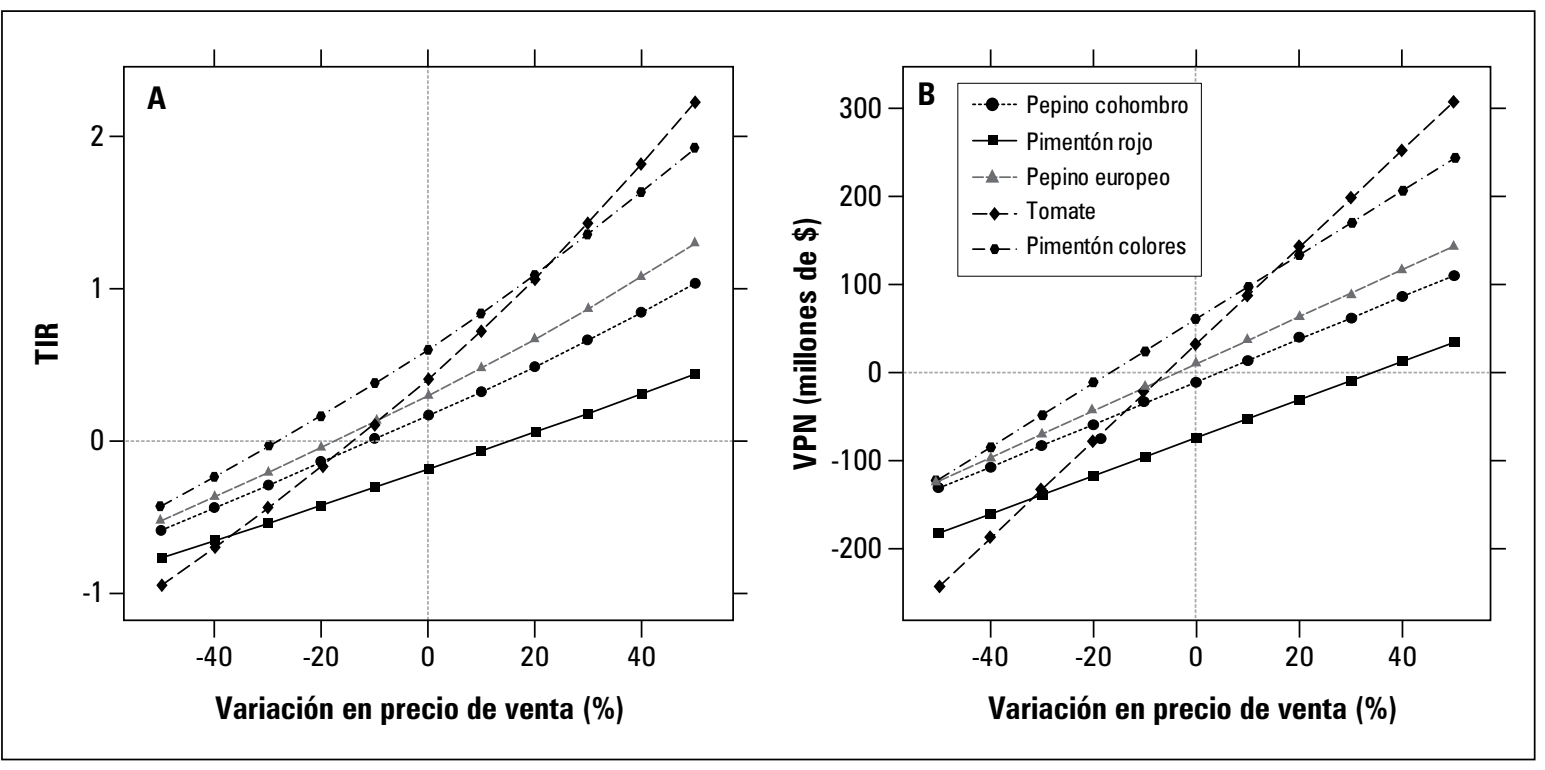

Figura 1. Efecto de la variación porcentual del precio pagado al productor de cinco proyectos productivos diseñados a 5 años y establecidos bajo invernadero sobre (A) la tasa interna de retorno (TIR) y (B) el valor presente neto (VPN). El precio de referencia (variación $=0 \%$ ) corresponde al precio promedio pagado al productor durante los últimos cinco años en la Corporación de Abastos de Bogotá (Corabastos) de acuerdo con los boletines del Sistema de Información de Precios del Sector Agropecuario (Sipsa).

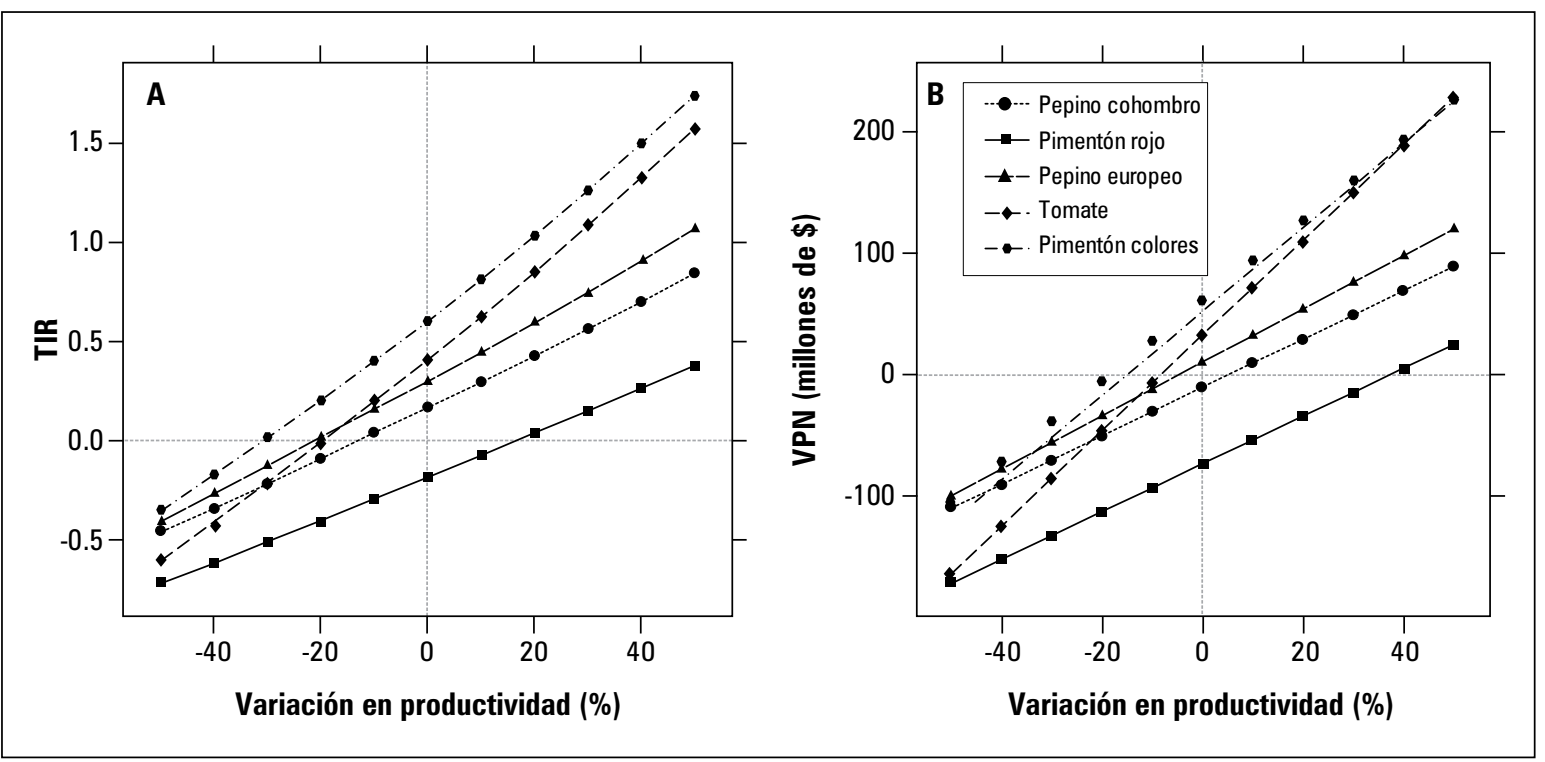

Figura 2. Efecto de la variación porcentual de la productividad de cinco proyectos productivos diseñados a 5 años y establecidos bajo invernadero sobre (A) la tasa interna de retorno (TIR) y (B) el valor presente neto (VPN). La productividad de referencia (variación $=0 \%$ ) corresponde a la productividad promedio obtenida a través de pruebas de campo (pepino y pimentón) o la reportada por productores mediante encuestas (tomate). 


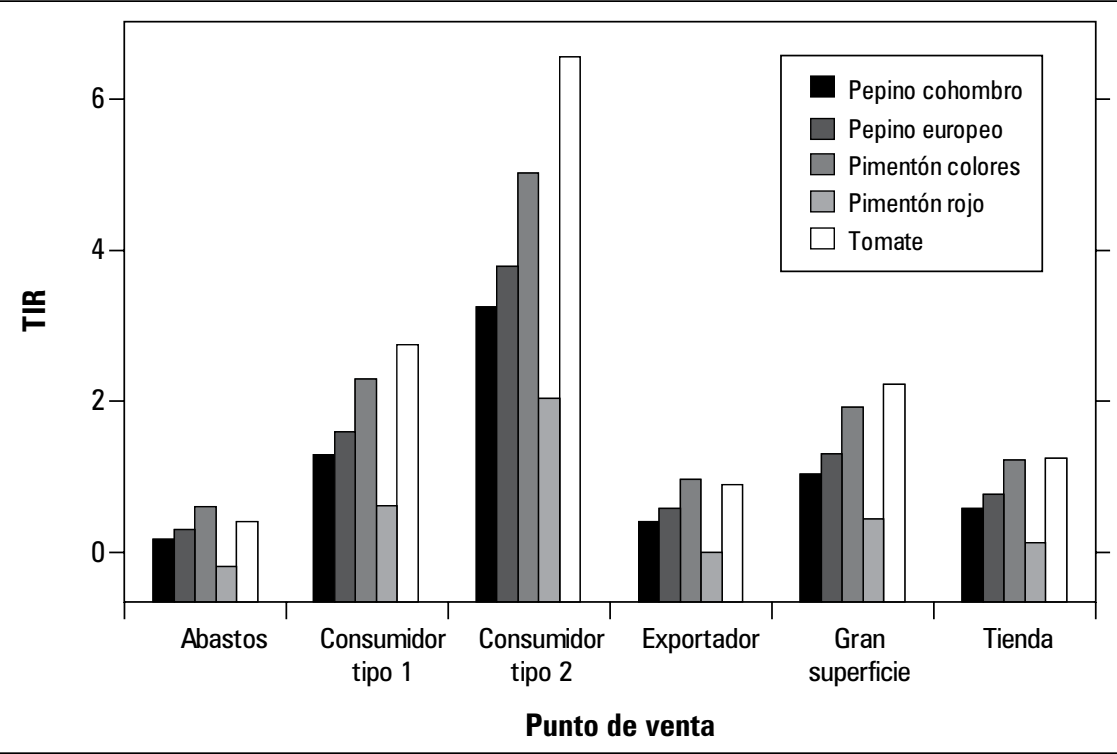

Figura 3. Efecto del precio pagado en diferentes puntos de venta de Bogotá de cinco cultivos establecidos bajo invernadero sobre la tasa interna de retorno (TIR) de proyectos diseñados a 5 años. Donde: consumidor tipo 1 (estrato $\leq 3$ ) y consumidor tipo 2 (estrato $>3$ )

rentes a las plazas mayoristas se pueden establecer proyectos viables y estables en el tiempo. Sin embargo, para realizar estos acuerdos comerciales, se deben tener en cuenta todos los aspectos característicos de cada tipo de cliente incluyendo sus condiciones y su método de comercialización (tabla 4).

Pozderek et al. (2010) realizaron una evaluación económica de proyectos productivos de pepino y pimentón tanto a campo abierto como bajo invernadero en Eslovenia. Específicamente bajo invernadero los proyectos de pepino y pimentón indicaron una relación $B / C$ promedio de 1,38 y 1,07 , respectivamente. Los resultados para este indicador financiero fueron similares a los obtenidos en el presente estudio. Las relaciones $B / C$ obtenidas para proyectos económicos de pepino y pimentón desarrollados en otros lugares fueron mucho mayores de acuerdo con las investigaciones ya citadas de Mohammadi y Omid (2010), Engindeniz y Gül (2009) y Gunadiet al. (2006). Sin embargo, es necesario tener en cuenta las particularidades de los sistemas de producción y de mercado en cada ubicación geográfica para poder contextualizar adecuadamente los resultados de cada estudio.

\section{CONCLUSIONES}

El establecimiento de especies como pepino y pimentón bajo condiciones de invernadero es viable económicamente solo si este va acompañado de una optimización de todo el sistema productivo. Los beneficios ofrecidos por el invernadero deben ser complementados por el productor mejorando sus prácticas de manejo (manejo cultural, fertilización, manejo de plagas y enfermedades) de manera que se reduzcan las limitantes biofísicas impuestas por el medio, logrando maximizar la productividad hasta donde sea posible. El cultivo del pimentón gourmet se muestra como una opción económicamente viable para diversificar o acompañar la producción del cultivo de tomate, pues presenta una mayor estabilidad en los precios de venta a lo largo del tiempo y genera una rentabilidad superior.

Es necesaria la búsqueda de nuevos canales de comercialización que generen una real estabilidad y mayores ingresos para el productor. Es así como el mercado de las tiendas, supermercados y plazas de barrio se muestran como clientes comerciales potenciales, ya que no tienen las exi- 
gencias de las grandes superficies ni manejan una volatilidad tan alta de precios como los centrales mayoristas de abastos. Las ventas al consumidor final mostraron el mejor escenario económico, sin embargo, la logística que se debe desarrollar para este tipo de mercado incluye un manejo es- pecial condicionado entre otros aspectos por la disponibilidad de transporte, el cumplimiento en las entregas, la optimización de rutas de entrega, las estrategias de mercadeo del producto y la consecución de una base de clientes mínima para que esta opción de mercado sea viable.

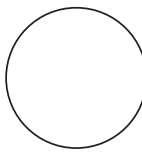

AGRADECIMIENTOS

El presente trabajo fue posible gracias a los apoyos recibidos por el Ministerio de Agricultura y Desarrollo Rural mediante la cofinanciación del proyecto "Desarrollo e implementación de un modelo de producción para los cultivos de pepino y pimentón bajo invernadero" (Código: 2008N62361-7013) y al Consejo Interuniversitario de Universidades Flamencas de Bélgica con su proyecto "Multidisciplinary assessment of efficiency and sustainability of smallholder-based tomato production systems in Colombia, with a roadmap for change" (Código: ZEIN2009PR364).

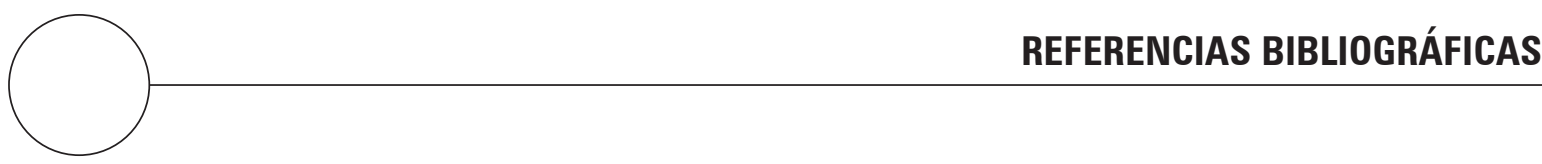

Arjona, H., M.L. Montalvo y M.A. Soto. 1992. Evaluación del comportamiento agronómico de tres híbridos y dos cultivares de pepino cohombro, Cucumis sativus L., bajo condiciones de invernadero en la Sabana de Bogotá. Revista Comalfi 19(1), 11-14.

Banco de la República de Colombia. 2011. Indicadores económicos. www.banrep.gov.co. consulta: marzo de 2011

Bojacá, C.R., R. Gil y E. Schrevens. 2011. The greenhouse effect in the high tropics of Colombia: a modeling approach. Acta Hort. 893, 791-796.

Bojacá, C.R., A. Cooman y H. Ubaque. 2009a. Ecofisiología del cultivo y manejo del clima. pp. 65-83. En: Escobar, H. (ed.). Producción de tomate bajo invernadero. Universidad de Bogotá Jorge Tadeo Lozano, Bogotá.

Bojacá, C.R., N.Y. Luque y O.I. Monsalve. 2009b. Análisis de la productividad del tomate en invernadero bajo diferentes manejos mediante modelos mixtos. Rev. Colomb. Cienc. Hortíc. 3(2), 188-198.

Bunte, F. 2009. Pricing in the greenhouse horticulture sector. Memorandum 09-026. Project code 40846.
LEI Wageningen UR, The Hague, The Netherlands.

Cámara de Comercio de Bogotá (CCB). 2011. Pasos para crear empresa. En: www.ccb.org.co; consulta: marzo de 2011.

Castilla, N. y J.I. Montero. 2008. Environmental control and crop production in Mediterranean greenhouses. Acta Hort. 797, 25-36.

Corporación Colombia Internacional (CCI). 2006. Canasta de productos del Plan Hortícola Nacional Tomate larga vida. pp. 437-461. En: Plan Hortícola Nacional. Corporación Colombia Internacional, Bogotá.

Dirección de Impuestos y Aduanas Nacionales (DIAN) 2011. Estatuto tributario. Www.dian.gov.co; consulta: marzo de 2011.

Engindeniz, S. y A. Gül. 2009. Economic analysis of soilless and soil-based greenhouse cucumber production in Turkey. Scientia Agricola 66(5), 606-614.

Escobar, H. 2009. Análisis económico. pp. 135-143. En: H. Escobar (ed.). Producción de tomate bajo inver- 
nadero. Universidad de Bogotá Jorge Tadeo Lozano, Bogotá.

Fondo para el Financiamiento del Sector Agropecuario (Finagro). 2011. ABC del agrocrédito. www.finagro.gov.co; consulta: marzo de 2011.

Gunadi, N., W. Adiyoda, T. Moekasan y A.P. Everaarts. 2007. Constraints and potential of sweet pepper cultivation in plastic houses in Indonesia. Acta Hort. 761, 305-312.

Jaramillo, J. 2009. The state of research in tomato in Colombia. Acta Hort. 821, 47-52.

Miranda, D., G. Fischer, J.C. Barrientos, C. Carranza, M. Rodríguez y O. Lanchero. 2009. Characterization of productive systems of tomato (Solanum lycopersicum L.) in producing zones of Colombia. Acta Hort. 821, 35-46.

Mohammadi, A. y M. Omid. 2010. Economical analysis and relation between energy inputs and yield of greenhouse cucumber production in Iran. Appl. Energy 87, 191-196.

Ozkan, B., A. Kurklu y H. Akcaoz. 2004. An input-output energy analysis in greenhouse vegetable production: a case study for Antalya region of Turkey. Biomass Bioenergy 26, 89-95.

Pozderek, S., K. Pažek y M. Bavec. 2010. Economics of peppers and salad cucumbers production on an open land and in a protected space. Agriculturae Conspectus Scientificus 75(3), 127-132.

R Development Core Team. 2011. R: A language and environment for statistical computing. R Foundation for Statistical Computing, Vienna, Austria. En: www.r-project.org; consulta: febrero de 2011.

Singh, B. y N.P.S. Sirohi. 2006. Protected cultivation of vegetables in India: problems and future prospects. Acta Hort. 710, 339-342. 